OPEN

SUBJECT AREAS:

ELECTRICAL AND

ELECTRONIC

ENGINEERING

APPLIED PHYSICS

Received

19 July 2013

Accepted

28 August 2013

Published

16 September 2013

Correspondence and requests for materials should be addressed to

T.S. (shimobaba@ faculty.chiba-u.jp)

\section{In-line digital holographic microscopy using a consumer scanner}

\author{
Tomoyoshi Shimobaba' ', Hiroya Yamanashi', Takashi Kakue', Minoru Oikawa', Naohisa Okada', \\ Yutaka Endo', Ryuji Hirayama', Nobuyuki Masuda² \& Tomoyoshi Ito'
}

'Graduate School of Engineering, Chiba University, 1-33 Yayoi-cho, Inege-ku, Chiba 263-8522, Japan, ${ }^{2}$ Faculty of Engineering, Nagaoka University of Technology, 1603-1 Kamitomioka, Nagaoka, Niigata 940-2188, Japan.

We demonstrate an in-line digital holographic microscopy using a consumer scanner. The consumer scanner can scan an image with $4,800 \mathrm{dpi}$. The pixel pitch is approximately $5.29 \mu \mathrm{m}$. The system using a consumer scanner has a simple structure, compared with synthetic aperture digital holography using a camera mounted on a two-dimensional moving stage. In this demonstration, we captured an in-line hologram with $23,602 \times 18,023$ pixels $(\approx 0.43$ gigapixels $)$. The physical size of the scanned hologram is approximately $124 \mathrm{~mm} \times 95 \mathrm{~mm}$. In addition, to accelerate the reconstruction time of the gigapixel hologram and decrease the amount of memory for the reconstruction, we applied the band-limited double-step Fresnel diffraction to the reconstruction.

D igital holographic microscopy (DHM) captures a hologram with an electronic device such as a CMOS or CCD camera, and the captured hologram is reconstructed on a computer using diffraction calculation ${ }^{1,2}$. In order to increase the field-of-view, lateral and depth resolving powers of the reconstructed image, we need to capture a large hologram, for example, the amount of gigapixels achieved in recent researches ${ }^{3-6}$. There are several methods available to capture a gigapixel hologram. In astronomy, a single CCD device with over 0.1 gigapixels has been achieved; however such a large area CCD is expensive. Another method for acquiring a gigapixel hologram is synthetic aperture digital holography using a camera mounted on a two-dimensional moving stage (or moving a reference light). Reference 5 reported a gigapixel digital holography using a typical color CCD that has Bayer color filter, and multiple holograms obtained by changing illumination angles of a reference light. A hologram captured with such a color CCD sensor and green light source lacks the pixels corresponding to the red and blue due to the Bayer filter, that is the effective pixel number is decreased. In order to increase the pixel number, Ref. 5 proposed ideal interpolation technique of the lacked pixels by using adjacent pixels. Recently, gigapixel microscopy using a consumer scanner has been proposed ${ }^{7}$. The approach is excellent because the microscopy has a wide field-of-view, low-cost and simple structure; however, it cannot observe a sample in the depth direction.

In this paper, we demonstrate an in-line DHM using a consumer scanner, inherently observing a sample in three-dimensions. In-line $\mathrm{DHM}^{8,9}$ is capable of obtaining a hologram without using beam splitters and mirrors. The consumer scanner can maximally scan an image with 4,800 dpi. The pixel pitch is approximately $5.29 \mu \mathrm{m}$. The DHM system using a consumer scanner has a simple structure, compared with synthetic aperture digital holography. In this demonstration, we captured an in-line hologram with $23,602 \times 18,023$ pixels $(\approx 0.43$ gigapixels). The physical size of the scanned hologram is approximately $124 \mathrm{~mm} \times 95 \mathrm{~mm}$. In addition, to accelerate the reconstruction time of the gigapixel hologram and decrease the memory usage for the reconstruction, we applied the band-limited double-step Fresnel diffraction (BL-DSF) ${ }^{10}$ to the reconstruction.

\section{Results}

We show a hologram of the USAF1951 test target captured by the DHM and the reconstructed image. Figure 1 shows the hologram with $23,602 \times 18,023$ pixels ( 0.43 gigapixels) and the hologram is sampled at about $5.29 \mu \mathrm{m}$. The hologram is captured under the condition that the distance between the scanner and the sample is $50 \mathrm{~cm}$ and the distance between the sample and the light source is $15 \mathrm{~cm}$. Because the number of pixels in the hologram is much larger than the resolution of a display $(1,920 \times 1,080)$, the decimated hologram by image processing is displayed. The inset shows the raw hologram in a part of the hologram (in the red square). We can observe the interference fringe of the hologram. Although the scanner can obtain a hologram maximally in 16bit/pixel, we 


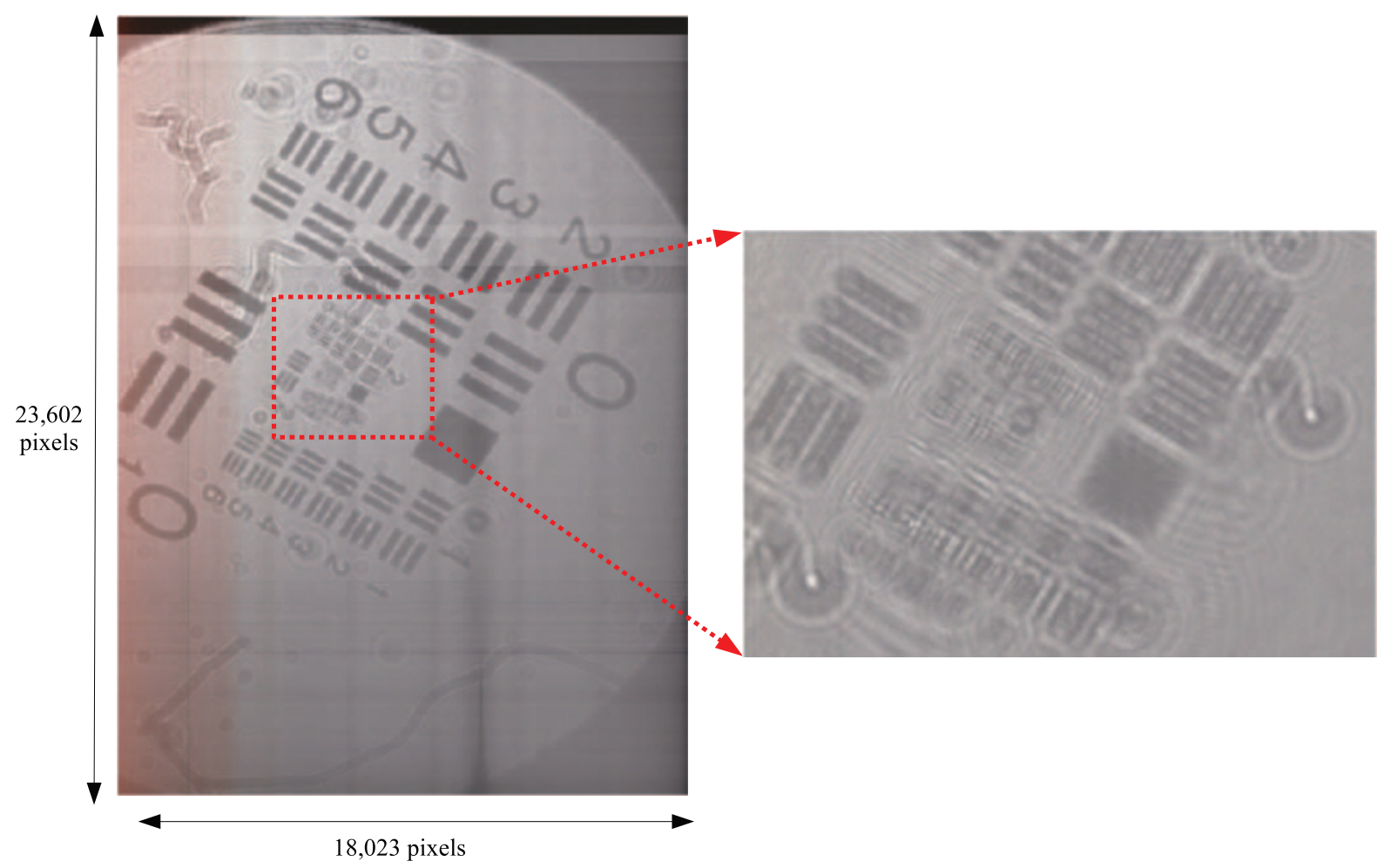

Figure $1 \mid$ Hologram of USAF1951 test target with 23, $602 \times 18,023$ pixels and the hologram is sampled at about $5.29 \mu \mathrm{m}$.

were unable to recognize the difference between the reconstructed images in 8 bit and 16bit/pixel. Therefore, we used a hologram captured by 8 bit/pixel in terms of the calculation time and the memory usage.

The reconstructed images are shown in Figure 2. The observational area of Fig. 2(a) is about $22 \times 29 \mathrm{~mm}^{2}$. Figure 2(b) shows the details of the red square in Fig. 2(a). Figure 2(c) shows the red square in Fig. 2(b) in greater detail. We can observe a reconstructed image in the resolving power of about $8.8 \mu \mathrm{m}$. The movie shows the observation of the reconstructed image as magnifying the image.

In a large hologram, the calculation time and memory usage for the reconstruction are important issues. We compared the calculation time and memory usage in the reconstruction of the hologram (Fig. 1) using the angular spectrum method (ASM) ${ }^{2}$ and BL-DSF ${ }^{10}$. In the calculation, we used Linux (64-bit) as the operating system and Intel Core i7-2600S as the CPU, the PC has the memory amount of 16 Gbytes. In ASM, the calculation time and the memory usage were about 355 seconds and 12.6 Gbytes, while the calculation time and the memory usage of BL-DSF were about 177 seconds and 3.2 Gbytes, respectively. The peak signal-to-noise ratio (PSNR) between the reconstructed images of ASM and BL-DSF is over $30 \mathrm{~dB}$. ASM is widely used in digital holography community as the reconstruction method. Therefore, we use reconstruction results obtained by ASM as the criteria to other reconstruction method, namely BL-DSF. Generally speaking, the difference of two images is very few when PSNR is over $30 \mathrm{~dB}$.

Figure 3 shows a hologram that, records an ant and water-flea placed at $30 \mathrm{~cm}$ and $50 \mathrm{~cm}$ from the scanner, respectively, and the reconstructed images. The number of pixels of the hologram is 23 , $602 \times 18,023$. When focusing on the water-flea, the ant is unfocused. While, when focusing on the ant, the water-flea is unfocused.

\section{Discussion}

Figure 4 shows the outline and photograph of the in-line DHM using a consumer scanner, respectively. In-line $\mathrm{DHM}^{8,9}$ is capable of obtaining a hologram without using beam splitters and mirrors. Samples are placed between a light source and the hologram. The diffracted and undiffracted lights by the samples are regarded as the object light and the reference light, respectively, then, the

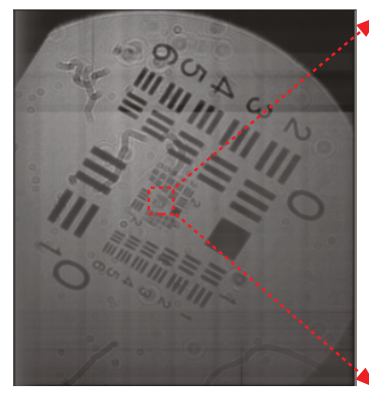

(a)

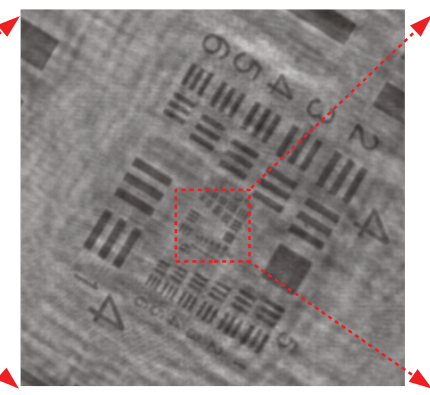

(b)

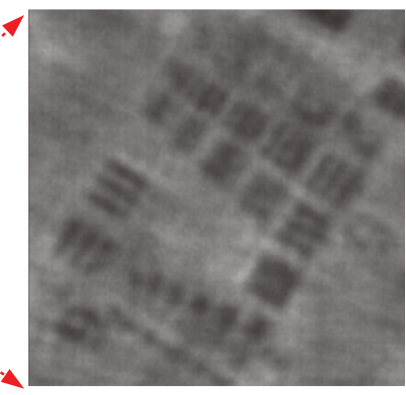

(c)

Figure $2 \mid$ Reconstructed images from the hologram of USAF1951 with $23,602 \times 18,023$ pixels. (See supplementary video). 


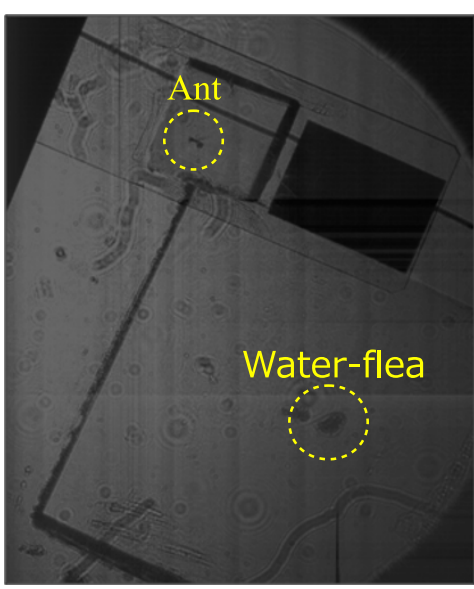

Hologram
Reconstructed image(Focus on water-flea)

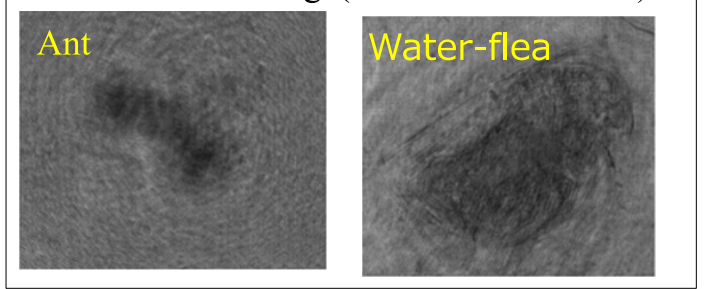

Reconstructed image(Focus on ant)
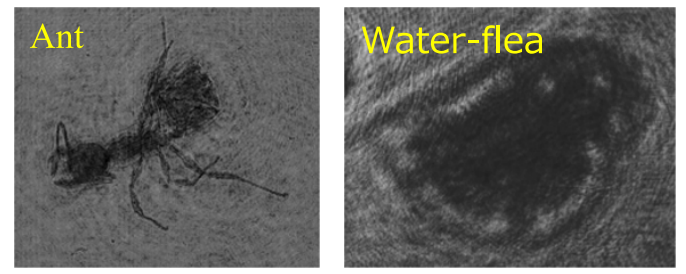

Figure $3 \mid$ Hologram that, records an ant and water-flea placed at $30 \mathrm{~cm}$ and $50 \mathrm{~cm}$ from the scanner, and the reconstructed images.

interference fringe of these lights generates a hologram. In-line DHM can control the area of the hologram and the magnification of the object light just by the location of the light source or the numerical aperture (NA) of an objective lens. If using a holographic setup that requires beam splitters, lens and mirrors, a large aperture is needed because the scanner has a large aperture; therefore, the in-line DHM is suitable for gigapixel holograms.

We used a fiber-output laser with a wavelength of $405 \mathrm{~nm}$. Although the output of the laser has a spherical wave, we used an objective lens with a magnification of $\times 10$ and an NA of 0.25 , to expand the angle of spread of the fiber-output laser. We placed samples between the objective lens and the scanner. The scanner captures an in-line hologram by moving the image sensor of the scanner.

As shown in Fig. 5, consumer scanners are mainly categorized by two types: "CCD" and "Contact Image Sensors (CIS)" scanners. Regarding CCD scanners (Fig. 5(a)), they have a two-dimensional CCD sensor whose size is smaller than the scan surface (cover glass); therefore, a reduction optical system composed of some mirrors and a lens is required to reduce the image on the scan surface to the CCD. And CCD sensors generally have a color filer for scanning color images; however, the color filter disturbs the capturing of holograms ${ }^{5}$.

In contrast, CIS scanners (Fig. 5(b)) have a simpler structure than CCD scanners because CIS sensors are one-dimensional devices whose size is the same as the scan surface; therefore, reduction of

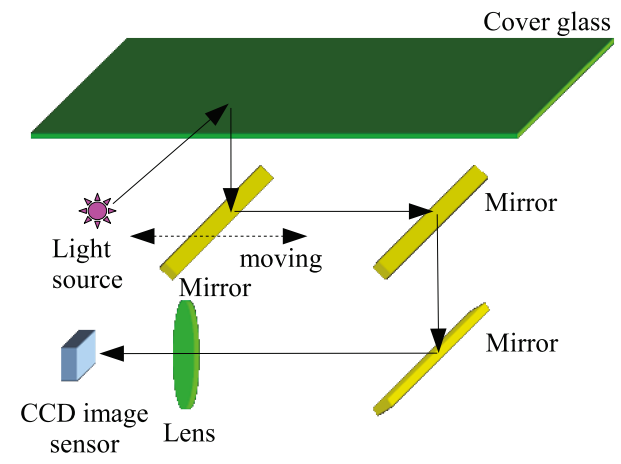

(a) CCD scanner the optical system in CCD scanners is not required. In addition, because CIS sensors are one dimensional, the electronic circuit is simpler than CCD sensors; therefore, the CIS scanners can capture an image with 16bit/pixel maximally, while most CCD scanners capture an image only with 8bit or 12bit/pixel maximally. CIS sensors also do not need a color filter unlike CCS scanner because CIS sensors capture a color image by switching RGB light sources in time-divisions; therefore, CIS scanners are not adversely affected by the color filter. Thus, we adopted a CIS scanner in this research because CIS scanners are suitable for hologram recording.

We used "CanoScan LiDE 210" made by Canon as the scanner. The scanner has a CIS image sensor and can capture an image with the same resolutions $(4,800 \mathrm{dpi})$ in the horizontal and vertical directions. We used "ScanGear (32-bit version)" as the scanner software. The GRIN lens attached to the CIS sensor is used for the imaging of scanning plane. However, the GRIN lens hamper a hologram recording. Therefore, we removed the GRIN lens. We covered the light source by a black tape because of cutting off the light. The scanner can maximally scan an A4 size image $(297 \mathrm{~mm} \times 210 \mathrm{~mm})$ with $4,800 \mathrm{dpi}$, theoretically achieving a resolution of $56,144 \times 39,698 \approx$ 2.22 gigapixels. However, we could capture a hologram with 23, 602 $\times 18,023$ pixels because we might use 32 -bit version of the software.

\section{Methods}

The reconstruction of gigapixel holograms is very time-consuming and requires huge memory usage. We adopted BL-DSF ${ }^{10}$, which is an effective way to obtain a

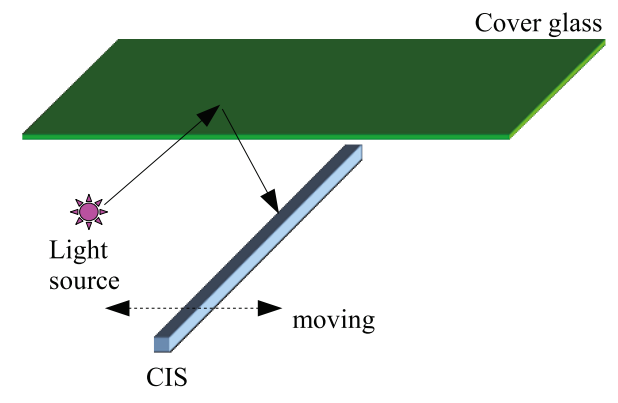

(b) CIS scanner

Figure $4 \mid$ In-line digital holographic microscopy using a consumer scanner. (Left) Outline of the system. (Right) Photograph of the system. 

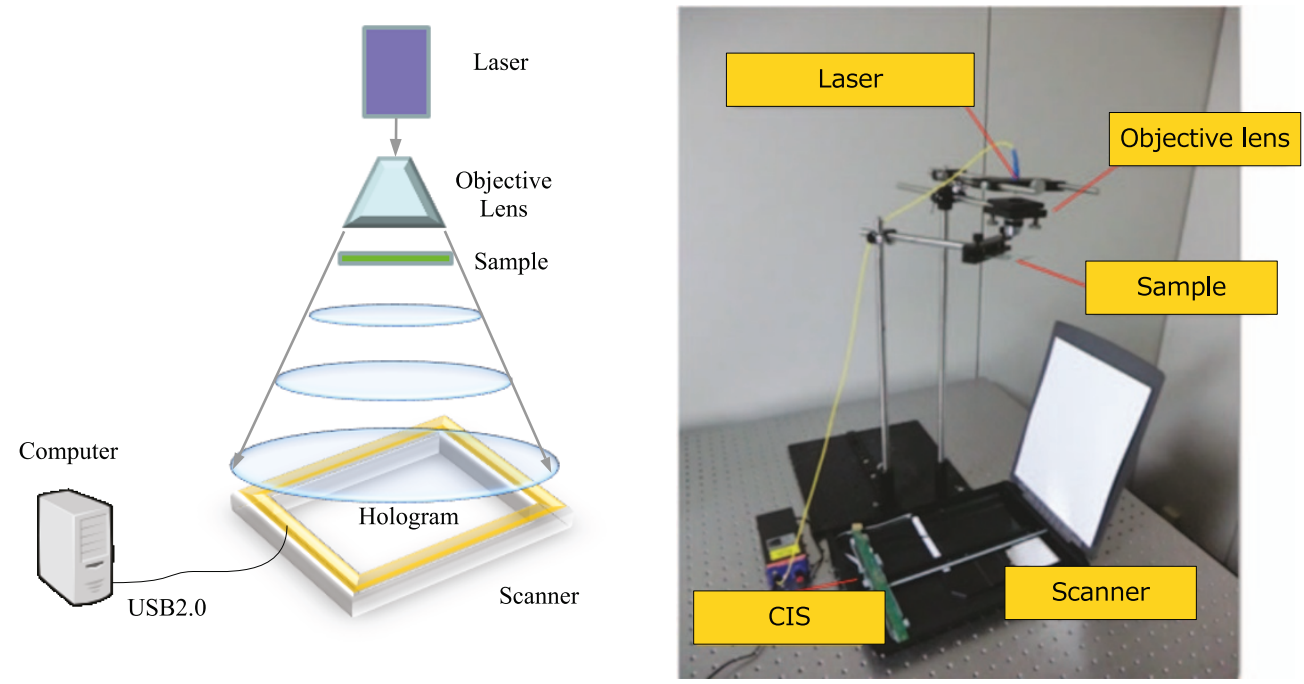

Figure 5| Two types of consumer scanners. (a) CCD scanner (b) CIS scanner.

reconstructed image from gigapixel holograms. In Fourier optics, diffraction calculations are categorized by two forms: the first is convolution-based diffraction and the second is Fourier transform-based diffraction. Here, we show ASM as an example of convolution-based diffraction. ASM is expressed as follows:

$$
u_{2}\left(x_{2}, y_{2}\right)=\mathrm{FFT}^{-1}\left[\operatorname{FFT}\left[u_{1}\left(x_{1}, y_{1}\right)\right] \exp \left(-2 \pi i z \sqrt{\left.1 / \lambda^{2}-f_{x}^{2}-f_{y}^{2}\right)}\right],\right.
$$

where $\lambda$ is the wavelength of light, the operators FFT $[\cdot]$ and $\mathrm{FFT}^{-1}[\cdot]$ are the fast Fourier and inverse fast Fourier transform respectively, $u_{1}\left(x_{1}, y_{1}\right)$ and $u_{2}\left(x_{2}, y_{2}\right)$ indicate a source and destination planes, $\left(f_{x}, f_{y}\right)$ is the coordinate on the frequency domain and $z$ is the propagation distance. The merit of convolution-based diffraction is that the sampling rates on the source and destination planes are the same; however, the demerit is the need to expand the source and destination planes by zero-padding to avoid noise. The form of ASM is the convolution using FFT. Generally speaking, the numerical calculation of the convolution using FFT that has circular property requires zero-padding to be the size of $2 N \times 2 N$, where $N$ is the horizontal and vertical pixel numbers of the hologram, during the calculation. If we do not use the zeropadding, the reconstructed image will incur noise by wraparound due to the circular property. The zero-padding can avoid the wraparound. The usage memory and calculation cost of ASM are proportional to $4 N^{2}$ and $4 N^{2} \log 4 N$, respectively. It takes large memory usage and long calculation time.

To overcome this problem, double-step Fresnel diffraction (DSF) has been proposed $^{11}$. It calculates the light propagation between the source to destination planes by twice the Fourier transform-based diffraction, via a virtual plane $\left(x_{v}, y_{v}\right)$. DSF does not need zero-padding because DSF is based on Fourier transform-based diffraction. In addition, although most Fourier transform-based diffractions change the sampling rates on the source and destination plane, DSF does not change them. Due to the original DSF incurring the aliasing noise under certain conditions, we proposed BLDSF introducing the rectangular function for band-limitation to the original DSF. BLDSF is expressed as follows:

$$
\begin{aligned}
& u_{2}\left(m_{2}, n_{2}\right)= C_{z_{2}} \operatorname{FFT}^{\operatorname{sgn}\left(z_{2}\right)}\left[\exp \left(\frac{i \pi z\left(x_{v}^{2}+y_{v}^{2}\right)}{\lambda z_{1} z_{2}}\right) \operatorname{Rect}\left(\frac{x_{v}}{x_{v}^{\max }}, \frac{y_{v}}{y_{v}^{\max }}\right)\right. \\
&\left.\mathrm{FFT}^{\operatorname{sgn}\left(z_{1}\right)}\left[u_{1}\left(m_{1}, n_{1}\right) \exp \left(\frac{i \pi\left(x_{1}^{2}+y_{1}^{2}\right)}{\lambda z_{1}}\right)\right]\right]
\end{aligned}
$$

where $z_{1}$ is the propagation distance from the source plane to the virtual plane, $z_{2}$ is the propagation distance from the virtual plane to the destination plane,

$C_{z_{2}}=\exp \left(\frac{i \pi}{\lambda z_{2}}\left(x_{2}^{2}+y_{2}^{2}\right)\right)$. The operator FFT ${ }^{\operatorname{sgn}(z)}$ means the forward FFT when the sign of $z$ is positive and the inverse type when it is negative. See Ref. 10 how to determine the band-limiting area $\left(x_{v}^{\max }, y_{v}^{\max }\right)$. The form of BL-DSF is not the convolution. The usage memory and calculation cost of BL-DSF are only proportional to $N^{2}$ and $N^{2} \log N$, respectively. BL-DSF was implemented in our open-source wave optics library, $\mathrm{CWO}++{ }^{12}$.
1. Schnars, U. \& Juptner, W. Direct recording of holograms by a CCD target and numerical Reconstruction. Appl. Opt. 33, 179-181 (1994).

2. Kim, M. K. Principles and techniques of digital holographic microscopy. SPIE Rev. 1, 018005 (2010)

3. Brady, D. J. \& Lim, S. Gigapixel holography. 2011 ICO International Conference on Information Photonics (IP) 1-2 (2011).

4. Fienup, J. R. \& Tippie, A. E. Gigapixel synthetic-aperture digital holography. Proc. SPIE 8122, 812203 (2011).

5. Isikman, S. O. et al. Giga-Pixel Lensfree Holographic Microscopy and Tomography Using Color Image Sensors. PLoS ONE 7, e45044 (2012).

6. Di, J. et al. High resolution digital holographic microscopy with a wide field of view based on a synthetic aperture technique and use of linear CCD scanning. Appl. Opt. 47, 5654-5659 (2008).

7. Zheng, G. et al. Gigapixel microscopy using a flatbed scanner. arXiv $\mathbf{1 2 0 9 . 4 0 4 5}$ (2012).

8. Sucerquia, J. G. et al. Digital in-line holographic microscopy. Appl. Opt. 45, 836-850 (2006)

9. Sucerquia, J. G. et al. Immersion digital in-line holographic microscopy. Opt. Lett. 31, 1211-1213 (2006)

10. Okada, N. et al. Band-limited double-step Fresnel diffraction and its application to computer generated hologram. Opt. Express 21, 9192-9197 (2013).

11. Zhang, F. et al. Algorithm for reconstruction of digital holograms with adjustable magnification. Opt. Lett. 29, 1668-1670 (2004).

12. Shimobaba, T. et al. Computational wave optics library for $\mathrm{C}++: \mathrm{CWO}++$ library. Comput. Phys. Commun. 183, 1124-1138 (2012).

\section{Acknowledgments}

This work is supported by Japan Society for the Promotion of Science (JSPS) KAKENHI (Grant-in-Aid for Scientific Research (C) 25330125) 2013, and KAKENHI (Grant-in-Aid for Scientific Research (A) 25240015) 2013.

\section{Author contributions}

T.S. and T.I. suggested this technique and wrote the main manuscript text. H.Y. prepared Figs. 1-4. N.M. prepared Fig. 5. H.Y., T.K., M.O., N.O., Y.E. and R.H. performed the experiments. All authors reviewed the manuscript.

\section{Additional information}

Supplementary information accompanies this paper at http://www.nature.com/ scientificreports

Competing financial interests: The authors declare no competing financial interests.

How to cite this article: Shimobaba, T. et al. In-line digital holographic microscopy using a consumer scanner. Sci. Rep. 3, 2664; DOI:10.1038/srep02664 (2013). 